\title{
Characterization of LPS and interferon- $\gamma$ triggered activation-induced cell death in N9 and primary microglial cells: induction of the mitochondrial gateway by nitric oxide
}

\author{
Cell Death and Differentiation (2007) 14, 183-186. doi:10.1038/sj.cdd.4401989; published online 16 June 2006
}

\section{Dear Editor,}

Microglia, the resident immune cells of the central nervous system, are normally quiescent but become activated after infection or injury. ${ }^{1}$ As activated microglia can promote both damage and protection, ${ }^{1}$ their numbers require strict regulation, in part by 'activation-induced cell death' (AICD). In view of the key participation of microglia in neurological disorders, ${ }^{2}$ knowledge of the molecular mechanism of AICD is important. Studies have shown that AICD induced by lipopolysaccharide (LPS) and interferon (IFN) $\gamma$ occurs via two pathways, one of them mediated via NO and the other via caspase-11..$^{3,4}$ Many aspects of this process, however, remain to be elucidated. We investigated AICD induced by LPS and IFN $\gamma$ in mouse N9 microglia ${ }^{5}$ and rat primary microglia.

Treatment of N9 cells with LPS $(100 \mathrm{ng} / \mathrm{ml})$ or with IFN $\gamma$ $(100 \mathrm{U} / \mathrm{ml})$ did not substantially affect their viability. However, the combination of the two (LPS/IFN $\gamma$ ) induced massive cell death, which was inhibited by the caspase inhibitor Q-VD$\mathrm{OPH}(20 \mu \mathrm{M})$ and the nitric oxide synthase (NOS) inhibitors $\mathrm{N}^{\mathrm{G}}$-monomethyl-L-arginine (L-NMMA) $(0.5 \mathrm{mM})$ and $\mathrm{N}^{\mathrm{G}}$ nitroL-arginine methyl ester (L-NAME) (1 mM) (Supplementary data, Figure S1). Examination of the effect of LPS/IFN $\gamma$ on the expression and activity of NOS and on the expression of caspase-11 in N9 cells revealed that inducible NOS (iNOS) and caspase-11 expression, as well as NOS activity, were stimulated in LPS/IFN $\gamma$-treated N9 cells (Supplementary data, Figure S1). Collectively, these results demonstrated that NO and caspases play an important part in AICD in N9 microglia and that N9 cells exhibit the typical features of microglial AICD observed in other microglia. . $^{3,4,6}$

Next, we identified the caspases that are activated in LPS/ IFN $\gamma$-treated microglia. Treatment of N9 cells with LPS/IFN $\gamma$ induced activation of caspase -3 and caspase-7. Activation of caspase-7 was indicated by a decrease in the amount of procaspase- 7 and the appearance of the active subunit (Figure 1a and Supplementary data, Figure S2a). This activation was preceded by an increase in procaspase-7 expression. Caspase-3 activation was indicated by the appearance of the active subunit after LPS/IFN $\gamma$ treatment. In contrast to procaspase-7, the expression of procaspase-3 was not induced (data not shown). Activation of these two caspases was accompanied by cleavage of their substrate poly (ADP-ribose) polymerase (PARP) (Figure 1a and
Supplementary data, Figure S2a). Also activated in LPS/ IFN $\gamma$-treated $\mathrm{N} 9$ cells was caspase-2, indicated by a decrease in procaspase-2 and the concomitant appearance of a $36-\mathrm{kDa}$ band (representing the $\mathrm{p} 19$ subunit bound to the prodomain) (Figure 1a and Supplementary data, Figure S2a). Activation of caspases-7, -3 , and -2 and cleavage of PARP also occurred in AICD of LPS/IFN $\gamma$-treated rat primary microglial cultures (Figure 1a, and Supplementary data, Figure S2b). We did not detect upregulation of procaspase-7 expression in these cells, probably because by that time point (18 $\mathrm{h}$ after treatment) procaspase-7 had been cleaved to generate its active subunits.

Among the important regulators of apoptosis are the proand antiapoptotic proteins of the Bcl-2 family, of which the proapoptotic multidomain proteins Bax and Bak are needed for apoptosis mediated by mitochondria. The BH3-only proteins Bid and Bim induce Bax and Bak activation. This activation process is associated with oligomerization of Bax and Bak and the subsequent formation of membrane pores that release apoptotic mediators such as cytochrome $c^{7}$ Thus, with the object of determining the role of Bcl-2 family proapoptotic proteins in microglial AICD, we examined the effect of LPS/IFN $\gamma$ treatment on their expression, distribution, and activation in microglial cells.

Immunoblot analysis revealed that in LPS/IFN $\gamma$-treated N9 cells the expression of Bax and Bak was unchanged after treatment (data not shown), whereas the expression of Bid, as well as of $B i m$ isoforms $\left(B i m_{L}\right.$ and $\left.B i m_{E L}\right)$, was increased (Figure1a and Supplementary data, Figure S2a). In some experiments we could also detect upregulation of the $\mathrm{Bim}_{\mathrm{S}}$ isoform. The increase in Bid expression was accompanied by the appearance of tBid (Figure1a and Supplementary data, Figure S2a). Similar results were obtained in LPS/IFN $\gamma$ treated rat primary microglia, except that at the time point tested (18 h after treatment) we did not detect upregulation of Bid expression, probably because by that time point Bid had been cleaved to generate tBid.

The subcellular distribution of these proapoptotic proteins in N9 cells was not affected by the treatment with LPS/IFN $\gamma$. Bak was present mainly in the heavy membrane (HM) fraction, Bax was present in both the cytosolic and the HM fractions, Bim isoforms were present in the HM fractions, and Bid was 

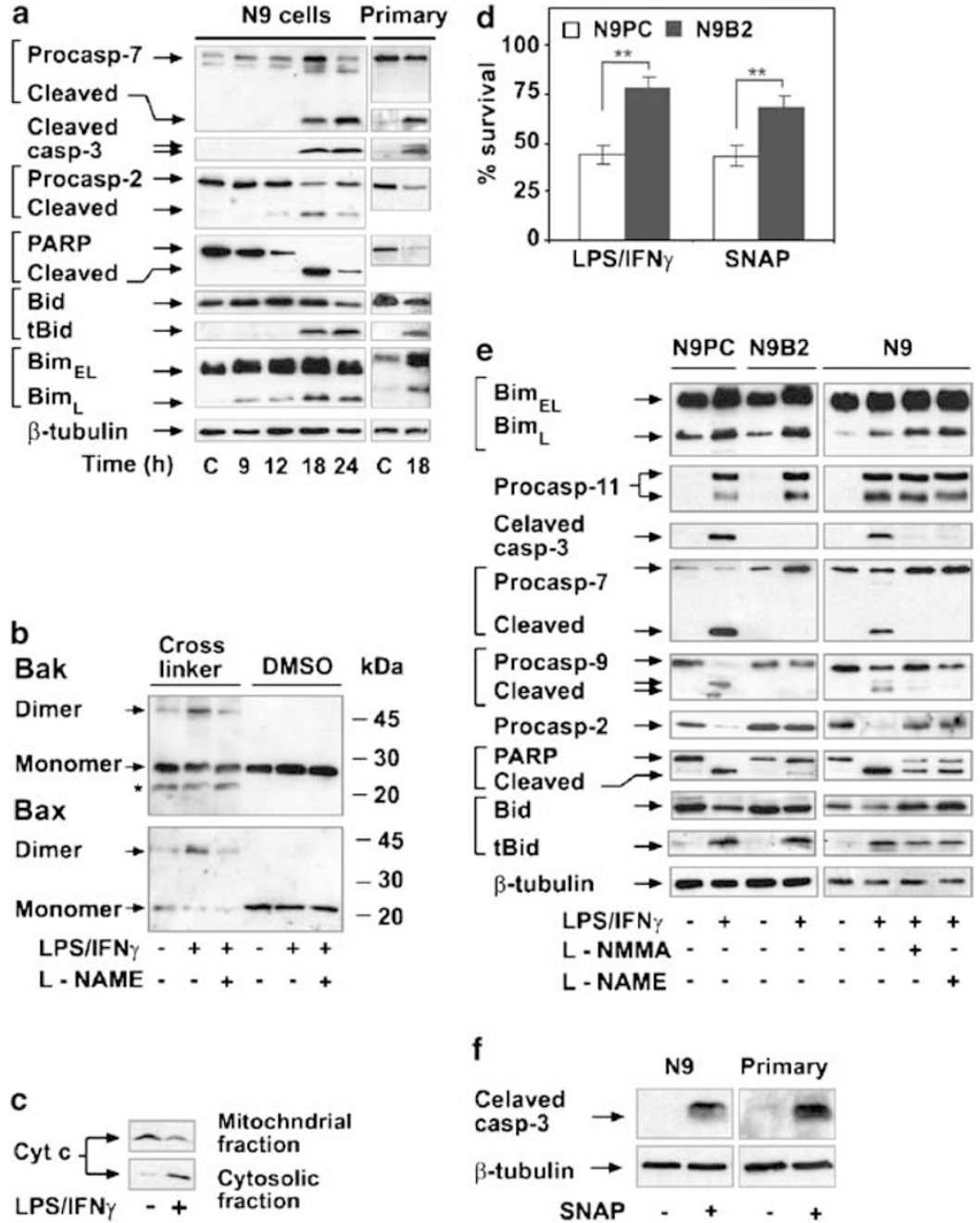

Figure 1 Apoptotic pathways in LPS/IFN $\gamma$-induced microglial AICD. N9 cells and rat microglial primary cultures (primary) were left untreated or were treated with LPS/ IFN $\gamma$. Primary microglia were prepared as previously described. ${ }^{8}$ Protein extracts were prepared from LPS/IFN $\gamma$-treated cells at the indicated times and from untreated cells (c) $24 \mathrm{~h}$ (N9 cells) and $18 \mathrm{~h}$ (primary cultures) from the start of the experiment. (a) Expression levels of caspase-7, active caspase-3, PARP, caspase-2, and Bid and Bim proteins were determined by immunoblot analysis as previously described. ${ }^{9}$ Membranes were reprobed with $\beta$-tubulin to verify the uniformity of sample loading Immunoblotting data are from a representative experiment $(n=3)$. (b) Bak and Bax oligomerization. N9 cells were treated without or with LPS/IFN $\gamma$ in the absence or presence of L-NAME $(1 \mathrm{mM})$ for $24 \mathrm{~h}$. Mitochondria-enriched heavy membrane fractions $(50 \mu \mathrm{g})$ prepared from the different treatments were incubated with BMH $(10 \mathrm{mM}$ ) (for Bak), DSS (10 mM) (for Bax), or the vehicle DMSO (5\%), and then lysed in RIPA buffer, cleared by centrifugation, and analyzed by immunoblotting with antiBak or anti-Bax Abs. Positions of the monomers and dimers of Bak and Bax are indicated. Monomeric Bak harboring an intramolecular cross-link is marked by an asterisk. Data shown are a representative experiment $(n=3)$. (c) Analysis of cytochrome clevels in cytosolic and mitochondrial subcellular fractions. N9 microglia were treated with LPS/IFN $\gamma$ for $9 \mathrm{~h}$. The cells were subjected to digitonin fractionation into cytosolic and mitochondrial fractions as described. ${ }^{10}$ The fractions were analyzed by immunoblotting with anti-cytochrome $c$. The data shown are from a representative experiment $(n=3)$. (d) Bcl-2 expression inhibits LPS/IFN $\gamma$ and NO-induced cell death. Stable Bcl-2-expressing (N9B2), empty vector-transfected (N9PC) cells were treated with LPS/IFN $\gamma$ or $0.5 \mathrm{mM}$ SNAP for 24 or $12 \mathrm{~h}$, respectively, or were left untreated. Cell viability was determined by the 3-(4,5-dimethylthiazol-2-yl)-2,5-diphenyl tetrazolium bromide (MTT) assay as previously described. ${ }^{11}$ Survival data were expressed as percentages of the MTT values without treatment. Values are means + S.D. $(n \geqslant 3) ;{ }^{*} P<0.01$, significantly different from untreated cells (Student's $t$-test). (e) Effects of Bcl-2 and NOS inhibitor on apoptotic proteins expression. N9PC, N9B2, or N9 cells were treated with LPS/IFN $\gamma$ in the absence or presence (1 $\mathrm{h}$ preincubation) of L-NMMA $(0.5 \mathrm{mM})$ or L-NAME $(1 \mathrm{mM})$ for $24 \mathrm{~h}$. Expression levels of Bim isoforms (Bim $\mathrm{EL}_{\mathrm{L}}$, and Bim $\mathrm{L}$ ), active caspase-3, $-7,-9,-2$, PARP, and Bid (and tBid) were determined by immunoblot analysis. Membranes were reprobed with $\beta$-tubulin. Immunoblotting data are from a representative experiment $(n=3)$. (f) NO induces caspase-3 activation. N9 microglia and primary cultures of rat microglia were left untreated or were treated with $1 \mathrm{mM}$ SNAP for $18 \mathrm{~h}$. Protein extracts were subjected to immunoblot analysis with the antiactive capase- 3 antibody. Membranes were reprobed with $\beta$-tubulin. Data shown are from a representative experiment $(n=3)$

present in the cytosol both before and after treatment. tBid, was found in both the cytosolic and the HM fractions (Supplementary data, Figure S3).

The finding that LPS/IFN $\gamma$ treatment led to an increase in the amount of tBid and Bim isoforms in the mitochondria suggested that these $\mathrm{BH}$-only proteins might cause activation of Bax and Bak. We therefore assessed Bax and Bak oligomerization by carrying out chemical cross-linking experiments. Intact mitochondria isolated from LPS/IFN $\gamma$-treated and untreated N9 cells were incubated with the cross-linking agent bis-maleimidohexane (BMH) (for Bak cross-linking) or disuccinimidyl suberate (DSS) (for Bax cross-linking). In the absence of LPS/IFN $\gamma$, Bax and Bak were detected mainly as monomers (Figure 1b). After LPS/IFN $\gamma$-treatment, however, 
we observed an increase in Bak (48 kDa) and Bax (42 kDa) homodimers (Figure 1b). Immunofluorescence staining analysis using anti-Bak $\mathrm{N}^{\prime}$-terminal (NT) or anti-Bax NT antibodies, which specifically recognize the NT-exposed conformation of Bax or Bak, revealed Bax/Bak NT exposure in LPS/IFN $\gamma$-treated N9 and primary rat microglial cells but not in untreated cells. Q-VD-OPH did not prevent this Bax/Bak NT exposure, suggesting that caspase activity is not needed for Bax or Bak activation (Supplementary data, Figure S4 a and b). Collectively, these results suggested that treatment with LPS/IFN $\gamma$ induces activation of Bax and Bak, and hence that AICD in microglia is mediated via Bak/Bax. Therefore, we next examined whether LPS/IFN $\gamma$ treatment induces cytochrome $c$ release in LPS/IFN $\gamma$-treated N9 cells. Figure 1c shows that treatment with LPS/IFN $\gamma$ led to the appearance of cytochrome $c$ in the cytosolic fraction, accompanied by a reduction in the amount of cytochrome $c$ in the mitochondrial fraction. Furthermore, cytochrome $c$ immunofluorescence staining revealed that some of the LPS/IFN $\gamma$-treated N9 microglia and primary rat microglia exhibited a diffuse cytochrome $c$ staining, which was not attenuated by treatment with Q-VD$\mathrm{OPH}$, whereas the untreated cells exhibited a perinuclear punctuated cytochrome $c$ staining pattern (Supplementary data, Figure S4c). The diffuse staining pattern of cytochrome $c$ and its appearance in the cytosolic fraction suggested that cytochrome $c$ is released during AICD of LPS/IFN $\gamma$-treated microglial cells, and therefore that the death process is preceded by generation of the apoptosome, with subsequent activation of caspase-9. This possibility was verified by the observed appearance of active caspase- 9 cleavage products in the immunoblots of LPS/IFN $\gamma$-treated N9 cells (Figure 1e).

The results presented thus far suggested that microglial AICD is mediated by activation of the mitochondrial gateway. To further assess the role of the mitochondrial pathway in microglial AICD, we examined whether this death would be inhibited by expression of $\mathrm{Bcl}-2$, which inhibits apoptosis mainly by inhibiting the mitochondrial pathway. N9 cell lines expressing Bcl-2 were generated and their sensitivity to LPS/ IFN $\gamma$ treatment was examined. The Bcl-2-expressing N9 cells (N9B2 cells) were strongly resistant to LPS/IFN $\gamma$ treatment, whereas the N9 cells transfected with empty vector (N9PC) cells were highly sensitive to it (Figure 1d). Next, we identified the step in the apoptotic cascade that is affected by Bcl-2 by examining NO production, induction of expression of apoptotic proteins, and activation of caspases in LPS/IFN $\gamma$-treated N9B2 cells. Bcl-2 expression did not prevent LPS/IFN $\gamma$ induced NO production (Supplementary data, Figure S5), nor did it prevent the LPS/IFN $\gamma$-induced increase in expression of Bim isoforms (Supplementary data, Figure S2c) and caspase11. However, it almost completely abolished caspase activation (caspases-9, -2, -3, and -7) and activity (cleavage of PARP) (Figure 1e). With regard procaspase-7, it was found to be upregulated, probably because it was not cleaved. Interestingly, expression of $\mathrm{Bcl}-2$ did not prevent the generation of tBid (Figure 1e), suggesting that most of the tBid is generated by caspases whose activation is independent of the mitochondrial gateway.

Having shown that the process of microglial AICD depends on NO production and is mediated via the mitochondrial-apoptosomal-caspase pathway, we proceeded to identify the step in the apoptotic cascade that is affected by NO. Assessment of the effects of the NOS inhibitors L-NMMA and L-NAME on LPS/IFN $\gamma$-induced apoptotic protein expression and on caspase activation in N9 cells revealed that neither inhibitor prevented the LPS/IFN $\gamma$ induced increase in expression of Bim isoforms or caspase11 , but both inhibitors abolished the generation of the active caspase-7 subunit (Figure 1e). With regard to procaspase-7, in the absence of the NOS inhibitors the amount of this procaspase in LPS/IFN $\gamma$-treated N9 cells was decreased whereas in cells treated with the NOS inhibitors it was increased, probably for the same reasons as in the $\mathrm{Bcl}-2$ overexpression experiments.

Taken together, these results suggested that NO does not regulate the expression of these apoptotic proteins, but rather acts on the mitochondrial-apoptosomal-caspase pathway. To substantiate this notion we examined the effects of the NOS inhibitors on the activation of Bak/Bax, caspase-3, -9, and -2, as well as on the cleavage of PARP in N9 cells. Crosslinking assay and immunoblot analysis showed that the NOS inhibitors abolished the LPS/IFN $\gamma$-stimulated Bak and Bax oligomerization (Figure 1b) and substantially attenuated the activation of caspase-3, -9 , and -2 and the cleavage of PARP (Figure 1e). These results thus suggested that NO acts at the step of Bax/Bak activation or upstream of it. The generation of tBid in N9 cells was only moderately inhibited by the NOS inhibitors (Figure 1e). Moreover, significantly more Bid was observed in N9 cells treated with NOS inhibitor than in untreated control N9 cells, suggesting that the inhibitors did not prevent the LPS/IFN $\gamma$-stimulated increase in Bid expression. These results thus suggested that $\mathrm{tBid}$ is generated mainly by an NO-independent pathway and that, as with Bim and caspase-7, the increased Bid expression is not regulated by NO. Collectively, the results suggested that $\mathrm{NO}$ acts downstream of the induction of proapoptotic protein expression, at the level of the mitochondrial gateway, where it induces Bax/Bak activation and the subsequent apoptosomalcaspase pathway in N9 cells.

To further substantiate this notion we examined whether direct application of NO also induces apoptosis in microglia via the mitochondrial pathway, and if so, whether such death is inhibited by $\mathrm{Bcl}-2$. N9 and rat primary microglial cells were treated with the NO donor S-nitroso- $\mathrm{N}$-acetyl-penicillamine (SNAP). As expected from previous studies, ${ }^{3,4}$ this treatment caused cell death in both microglial cell systems. Death was accompanied by Bax and Bak activation (NT exposure (Supplementary data, Figure S6) and activation of caspase3 (Figure 1f)), suggesting that $\mathrm{NO}$ induces microglial apoptosis via the mitochondrial pathway.

To further investigate whether the apoptotic pathways induced by the NO donor and by LPS/IFN $\gamma$ are similar, we examined whether expression of Bcl-2, which inhibits cell death induced by LPS/IFN $\gamma$, would also inhibit the cell death induced by SNAP. As shown in Figure 1d, the SNAP-treated N9B2 cells were significantly more viable than the SNAPtreated N9PC cells. These results, together with those showing that overexpression of $\mathrm{Bcl}-2$ and treatment with NOS inhibitors exert similar inhibitory effects on LPS/IFN $\gamma$ induced cell death, imply that the apoptotic pathways induced by LPS/IFN $\gamma$ and by $\mathrm{NO}$ are similar. 
In summary microglial AICD is accompanied by upregulated expression of pro-apoptotic proteins and is mediated mainly via NO-promoted Bak/Bax activation and the mitochondrial gateway.

Acknowledgements. We thank Dr. Thomas Möller for helpful advices. This work was supported by the Israel Science Foundation and the Adams SuperCenter for Brain Research.

\author{
$L_{\text {Mayo }}{ }^{\text {and } R}$ Stein $^{\star, 1}$ \\ 1 Department of Neurobiochemistry, George S. Wise Faculty of Life Sciences, \\ Tel Aviv University, 69978, Ramat Aviv, Israel
}



* Corresponding author: R Stein, Department of Neurobiochemistry, George S. Tel: + 97236408608 ; Fax: + 97236407643

E-mail: reuvens@post.tau.ac.il

1. Streit WJ et al. (2005) Neurol Res 27: 685-691.

2. Mrak RE, Griffin WS (2005) Neurobiol Aging 26: 349-354.

3. Lee J et al. (2001) J Biol Chem 276: 32956-32965.

8. Weydt P et al. (2004) Glia 48: 179-182.

11. Lindenboim L et al. (1995) J Neurochem 64: 1054-1063.

\section{Compensatory mechanisms regulate the Bcl-2 rheostat and lymphocyte survival in the absence of $\mathrm{AKT} 1 / \mathrm{PKB} \alpha$}

Cell Death and Differentiation (2007) 14, 186-189. doi:10.1038/sj.cdd.4402000; published online 16 June 2006

\begin{abstract}
Dear Editor,
The serine-threonine protein kinase AKT (PKB) plays a central role in controlling essential cellular processes, including proliferation, glucose metabolism, angiogenesis and survival (for a review see Datta et al. ${ }^{2}$ ). Three major isoforms, $\mathrm{AKT} 1 / \mathrm{PKB} \alpha, \mathrm{AKT} 2 / \mathrm{PKB} \beta$ and $\mathrm{AKT} 3 / \mathrm{PKB} \gamma$, encoded by independent genes exist in mammals. All three isoforms are ubiquitously expressed although the levels of expression vary among tissues and AKT1 appears to be the predominant isoform. ${ }^{2}$ Studies in knockout mice lacking individual genes implicate specific, only partially overlapping functions for the different AKT isoforms. Mice lacking akt1 exhibit impaired growth with a reduction in body weight of $30 \%$ and a proportional decrease in the sizes of all major organs, identifying AKT1 as a key regulator of organismal growth. ${ }^{3,4}$ Thymocytes and mouse embryonic fibroblasts (MEF) derived from these animals were reported to display an increased susceptibility to spontaneous and stress-induced apoptosis. ${ }^{4}$ By contrast, akt2-deficient mice exhibit insulin resistance and a diabetic phenotype but are only mildly growth retarded. ${ }^{5}$ AKT2, therefore, is the relevant isoform controlling insulin regulated glucose metabolism. So far, apoptosis defects have not been reported in cells derived from mice lacking either akt2 or akt3. ${ }^{5-7} A k t 1$ and akt1/2 double deficient MEF, transformed by a dominant-negative version of p53 succumbed to cell death with identical kinetics when treated with the hexokinase inhibitor clotrimazole, indicating that
\end{abstract}

loss of akt2 does not further increase the apoptosis susceptibility of akt $1^{-1-}$ cells. $^{8}$

AKT-mediated signals have been reported to suppress apoptosis by modulating the expression and/or function of a series of pro- and antiapoptotic genes, many of which belonging to the $\mathrm{Bcl}-2$ family. ${ }^{9}$ In line with these observations, loss of $b c l-2, m c l-1$ or $b c l-X_{L}$ in mice causes depletion of lymphocytes, myeloid cells and erythroid progenitors, respectively, due to increased susceptibility of these cells to trophic factor deprivation. ${ }^{10}$ As loss of akt1 was reported to render thymocytes and primary MEF more susceptible to spontaneous as well as stress-induced cell death triggered by serum deprivation, glucocorticoids and $\gamma$-irradiation, ${ }^{4}$ we reasoned that the molecular basis for this phenomenon may be deregulated expression and/or function of certain pro- and antiapoptotic Bcl-2 family members, either repressed (e.g. Bad, Bim, or PUMA ${ }^{1,11,12}$ ) or induced (e.g. Bcl- $\mathrm{X}_{\mathrm{L}}$ or Mcl$1^{13,14}$ ) by AKT.

To explore this possibility, we analysed mRNA expression levels of pro-and antiapoptotic Bcl-2 family members in wt thymocytes or those lacking akt1. By applying quantitative real-time PCR analysis, we observed that the absence of akt1 does not impair the basal mRNA transcription of any known Bcl-2 family member in primary thymocytes. Neither an upregulation of proposed AKT-downstream targets of the proapoptotic Bcl-2 family members such as bim or puma nor a 